\title{
O PAPEL DA CONFIANÇA NA MARCA NA INTENÇÃO DE ADOÇÃO DE NOVAS TECNOLOGIAS
}

\author{
Mellina da Silva Terres \\ Doutoranda em Administração pela Universidade Federal do Rio Grande do Sul - UFRGS \\ mellinaterres@hotmail.com
}

\section{Clara Isabel Koetz}

Doutoranda em Marketing pela Universidade Federal do Rio Grande do Sul - UFRGS clara_koetz@uol.com.br

\section{Cristiane Pizzutti dos Santos}

Doutora em Administração pela Universidade Federal do Rio Grande do Sul - UFRGS

Professora da Universidade Federal do Rio Grande do Sul - UFRGS

crispizzutti@gmail.com

\section{Carla Schwengber ten Caten}

Doutora em Engenharia de Minas, Metalúrgica e de Materiais pela Universidade Federal do Rio Grande do Sul - UFRGS

Professora da Universidade Federal do Rio Grande do Sul - UFRGS

carlacaten@gmail.com

\section{RESUMO}

O processo de adoção de novas tecnologias é marcado pela assimetria de informações, no qual a empresa tem pleno conhecimento das suas características, mas o usuário a quem esta tecnologia se destina, de forma distinta, tem acesso a poucas informações. A fim de minimizar esta assimetria de informações, e com isto a incerteza e o risco inerentes a estes processos, as empresas podem investir em marcas fortes e confiáveis, que transmitam informações acerca da qualidade dos produtos para os consumidores. Neste trabalho tem-se por objetivo analisar a influência da confiança na marca, na intenção de adoção de novas tecnologias em trocas business-to-consumer. Para tal, foi aplicado o modelo Unified Theory of Acceptance and Use of Techonology (UTAUT), proposto por Venkatesh et al. (2003). A confiança na marca foi mensurada por meio da escala desenvolvida e validada por Delgado-Ballester et al. (2003), que abrange duas facetas: a confiança baseada na confiabilidade e a baseada nas intenções da marca. Os dados foram coletados através de uma survey com 348 respondentes e a análise dos dados foi realizada por meio de Modelagem de Equações Estruturais. Os resultados demonstraram que a confiança baseada nas intenções da marca, que está relacionada com a expectativa do consumidor acerca do comportamento futuro da empresa em resolver problemas associados ao consumo do produto, tem influência significativa na intenção do consumidor em adotar nova tecnologia. Além disso, o impacto dos construtos anteriormente inseridos no modelo UTAUT - a expectativa de desempenho, a expectativa de esforço e a influência social - na intenção do consumidor em adotar uma nova tecnologia foi confirmado.

Palavras-chave: Confiança na marca; Modelo UTAUT; Comportamento do consumidor; Novas tecnologias. 


\section{INTRODUÇÃO}

Os fatores que influenciam na adoção de novas tecnologias têm sido amplamente investigados por pesquisadores da área de sistemas de informação. Venkatesh et al. (2003) destacam que a pesquisa deste tema tem resultado em modelos teóricos distintos, fundamentados em áreas como sistemas de informação, psicologia e sociologia. No campo do marketing, um dos modelos que apresentou maior repercussão foi o Theory of Reasoned Action (TRA), que aborda o papel das influências motivacionais no comportamento do consumidor (Singh, Fassot, Chao, \& Hoffmann, 2006). Com o advento da internet, alguns estudos se basearam em modelos de aceitação de tecnologia a fim de compreender os fatores que influenciam o consumidor em relação ao comércio eletrônico (Pavlou, 2003; Singh et al., 2006; Njite \& Parsa, 2005). Singh et al. (2006), por exemplo, aplicaram o Technology Acceptance Model (TAM), na análise da aceitação do consumidor em relação a web sites internacionais.

A adoção de novas tecnologias pode ser definida como processo composto por uma série de ações e escolhas, ao longo do tempo, que permite a um indivíduo ou empresa avaliar uma ideia nova e optar por incorporá-la ou não às suas práticas correntes (Rogers, 1995). O elemento de inovação presente na tecnologia e, por conseguinte, o desconhecimento do indivíduo em relação a sua utilização e desempenho, estimula o risco percebido e interfere na decisão do consumidor. De fato, este processo ocorre em um contexto de assimetria de informações, no qual a empresa fabricante tem pleno conhecimento das características da tecnologia que está sendo disponibilizada, mas o usuário a quem esta tecnologia se destina, de forma distinta, tem acesso a poucas informações. Um dos problemas advindos da assimetria de informações é a seleção adversa, que ocorre quando a parte menos informada - no caso o futuro usuário - faz uma negociação pouco vantajosa, adquirindo um produto ou serviço que não corresponde às suas expectativas, justamente em virtude da pouca informação disponível (Molho, 1997). A fim de reduzir a assimetria de informações e minimizar o problema da seleção adversa, a empresa, que nessa situação caracteriza a parte mais informada, pode emitir sinais ao futuro usuário, os quais terão a função de transmitir informações, possibilitando uma negociação mais vantajosa para ambas as partes.

Nesse sentido, o desenvolvimento de marcas fortes e confiáveis pode exercer a importante função de sinalizar a qualidade da empresa e do produto para os consumidores, reduzindo a incerteza e o risco inerentes aos processos de adoção de novas tecnologias e influenciando positivamente as transações. A sinalização da qualidade por meio da marca tem sido abordada em diversos artigos (e.g., Erdem \& Swait, 1998; Erdem, Swait, \& Valenzuela, 2006), cuja ênfase está no papel que a marca exerce

Revista de Administração e Inovação, São Paulo, v. 7, n. 4, p.162-185, out./dez. 2010 
na redução da incerteza presente em contextos de assimetria de informação. A marca, por meio de seu conteúdo e credibilidade, emite sinais acerca da posição e confiabilidade da empresa e do produto, aumentando a qualidade percebida e diminuindo os custos informacionais e os riscos percebidos pelos consumidores (Erdem \& Swait, 1998). Assim, a marca consiste em um elemento de transmissão de um conjunto de características e de valores que representam a reputação conquistada por uma empresa ao longo de sua existência, estabelecendo uma situação de confiança que traz, na sua essência, a redução do risco percebido pelo consumidor.

Alguns estudos acerca da aceitação de novas tecnologias têm abordado a confiança (e.g., Njite \& Parsa, 2005; Lee, Ang, \& Dubelaar, 2005), bem como a relação entre risco e confiança (e.g., Pavlou, 2003) no comportamento do consumidor em relação ao comércio eletrônico. A ideia subjacente a estes artigos é que o papel da confiança torna-se elevado devido ao alto nível de incerteza e de risco existentes na maioria das transações online. Porém, o papel que a confiança na marca desempenha nos processos de adoção de novas tecnologias ainda permanece inexplorado na literatura. O objetivo deste trabalho está justamente em preencher esta lacuna; assim, pretende-se demonstrar que o desenvolvimento de marcas fortes e confiáveis pode exercer a importante função de reduzir o risco percebido e influenciar positivamente a intenção dos consumidores em adotar novas tecnologias.

Dessa forma, a principal contribuição deste estudo está em analisar a adoção de novas tecnologias, a partir de um olhar fundamentado na economia da informação, considerando que a confiança na marca pode ser um sinal que transmite informações acerca do produto e, dessa forma, possibilita a redução da incerteza e do risco intrínsecos à transação. Para tal, será aplicado o modelo Unified Theory of Acceptance and Use of Techonology (UTAUT), proposto por Venkatesh, Morris, Davis e Davis (2003), o qual foi originalmente formulado para analisar a adoção de tecnologias da informação em ambientes organizacionais, tendo sido adaptado para trocas business-to-consumer. A escolha do UTAUT deve-se ao fato de ter sido formulado de forma a contemplar os oito principais modelos de aceitação de tecnologia, tendo demonstrado os melhores resultados em relação a cada modelo, como o R2 ajustado, de 69\% e 70\% em duas fases distintas da pesquisa (Venkatesh et al., 2003). Assim, a adaptação e a aplicação do modelo UTAUT ao contexto business-to-consumer também representam uma importante contribuição deste estudo.

O presente trabalho está organizado da seguinte forma: na seção dois, são apresentados os fundamentos teóricos, os quais contemplam a confiança, confiança na marca e o modelo UTAUT de aceitação de tecnologias da informação e que apresentam, ao longo do seu desenvolvimento, a definição das hipóteses. Na seção três, os procedimentos metodológicos utilizados na condução da pesquisa são 
abordados. Na seção quatro, os resultados são discutidos e, posteriormente, na seção cinco, as considerações finais são desenvolvidas, as implicações acadêmicas e gerenciais são apresentadas e, finalmente, as sugestões para novas pesquisas são indicadas.

\section{FUNDAMENTAÇÃO TEÓRICA}

A fim de analisar o papel da confiança na marca na adoção de novas tecnologias é necessário compreender, primeiramente, a confiança de uma forma mais ampla, especificamente no tocante à sua relação intrínseca com o risco e com a redução da incerteza e, por conseguinte, examinar, mais especificamente, a confiança na marca. Além disso, é fundamental que se busque um entendimento mais aprofundado das dimensões da intenção de adoção de novas tecnologias. Estes tópicos serão abordados a seguir.

\subsection{CONFIANÇA}

A confiança tem sido abordada em diversas áreas como a sociologia, a psicologia e a antropologia, podendo ser definida como uma atitude frente à incerteza, à complexidade e à incapacidade de prever o futuro, ocorrendo numa estrutura de interação na qual é influenciada tanto pela personalidade quanto pelo sistema social, não podendo ser de forma exclusiva associada a um deles (Luhmann, 1979). Assim, diversos autores têm apresentado conceitos acerca da confiança (e.g., Crosby, Evans, \& Cowles, 1990; Mishra, 1996; Hagen \& Choe, 1998), os quais geralmente abordam questões relacionadas à expectativa de cumprimento de promessas (Barber, 1983; Hagen \& Choe, 1998) crença no acontecimento de atitudes favoráveis (Crosby, Evans, \& Cowle, 1990; Swan, Trawick, \& Silva, 1985) e disposição em aceitar um parceiro devido aos riscos envolvidos (Zand, 1972; Lewis \& Wiegert, 1985; Mishra, 1996).

Rousseau, Sitkin, Burt e Camerer (1998) analisaram a confiança de maneira interdisciplinar, identificando os pontos em comum e as principais divergências na forma como o tema é considerado em diferentes campos de estudo. Segundo eles, os economistas tendem a abordar a confiança de maneira calculista e institucional; psicólogos avaliam o tema de modo a enfocar nas características das pessoas em relacionamentos que envolvem confiança, em especial nos atributos cognitivos destas pessoas; e os sociólogos analisam a estrutura social e as propriedades nas quais estes relacionamentos de confiança

Revista de Administração e Inovação, São Paulo, v. 7, n. 4, p.162-185, out./dez. 2010 
estão inseridos. Os autores apresentam um conceito que consideram amplo, cujo ponto principal está em estabelecer o tema confiança, de maneira a contemplar as características propostas pelos autores destes diversos campos. Assim, confiança é definida como "[...] um estado psicológico que compreende a intenção de aceitar a vulnerabilidade baseada em expectativas positivas acerca das intenções de outra pessoa" (Rousseau et al., 1998, p. 395).

O fato do conceito de confiança ser associado ao risco e à relação de interdependência existente entre ambos faz com que este tema seja recorrentemente abordado na literatura. De acordo com Molm, Takahashi e Peterson (2000), o risco é uma condição necessária para o desenvolvimento da confiança em situações nas quais o indivíduo depende do comportamento do parceiro. Morgan e Hunt (1994) destacam a importância da confiança para o comprometimento, pois ela permite ao indivíduo visualizar uma situação de alto risco como sendo prudente, já que ele irá acreditar que seu parceiro não agirá de maneira oportunista. Segundo Rousseau et al. (1998), o risco é considerado essencial para o desenvolvimento da confiança nas abordagens econômica, psicológica e sociológica, sendo associado à possibilidade percebida de perda. Assim, a relação de interdependência entre risco e confiança ocorre de forma recíproca: o risco cria a oportunidade da confiança; esta, por sua vez, conduz a pessoa a arcar com a tomada de risco. Na essência do risco encontra-se a questão da incerteza; dessa forma, a confiança não ocorreria se os relacionamentos estivessem inseridos em contextos de completa certeza e sem risco.

Delgado-Ballester, Munuera-Aleman e Yague-Guillen (2003) argumentam que o risco percebido é uma das condições para que a confiança se estabeleça, ou seja, os consumidores irão utilizar a confiança na marca como um critério de decisão determinante nas suas compras em situações em que enfrentarem níveis de incerteza relacionados à satisfação das suas expectativas de consumo. A confiança na marca será abordada no subitem a seguir.

\subsubsection{CONFIANÇA NA MARCA}

A confiança na marca, da mesma forma que o tema confiança, tem sido alvo de diversas disciplinas, como economia, psicologia e sociologia, além de áreas aplicadas como gestão e marketing. Esta interdisciplinaridade, que por um lado adiciona riqueza ao tema, por outro, torna difícil a integração das diferentes abordagens e de que seja encontrado um consenso no que tange a sua natureza (DelgadoBalester \& Munuera-Alemán, 2005). Assim, uma das principais linhas de estudo acerca da confiança na marca se baseia em uma abordagem psicológica, cujo fundamento está na expectativa do consumidor acerca da confiabilidade da marca. A essência desta abordagem consiste na crença que os consumidores 
têm em relação a qualidades específicas que a marca possui e que a fazem consistente, como competência, honestidade e responsabilidade (Delgado-Balester \& Munuera-Alemán, 2005). Esta linha de estudo é contemplada por autores como Chaudhuri e Holbrook (2001) e Delgado-Ballester (2004), entre outros.

Delgado-Ballester (2004) propõe um conceito que busca abranger as diversas áreas de estudo, como psicologia social, sociologia, economia, gestão e marketing, segundo o qual, a confiança na marca envolve a confiabilidade da marca (brand reliability) e as intenções em relação à marca (brand intentions). A marca, nessa perspectiva, adquire o caráter de um parceiro relacional ativo, que personifica características de uma pessoa com a qual o consumidor estabelecerá um relacionamento. A seguir, cada uma destas dimensões será analisada.

A confiança baseada na credibilidade da marca envolve as expectativas e necessidades dos consumidores e o quanto eles acreditam que a marca estará apta a satisfazê-los. Este tipo de confiança está relacionado, em sua essência, aos aspectos técnicos e baseados em competências que a marca dispõe. Assim, a confiabilidade de uma marca é essencial para que a confiança na marca se estabeleça, pois o fato da marca de fato atingir a promessa que ela representa para o consumidor faz com que ele se torne mais confiante em relação à ocorrência de satisfação futura. Ou seja, subjacente a esta dimensão existe um senso de previsibilidade de que a marca satisfaz as necessidades de consumidor de diversas maneiras positivas (Delgado-Ballester, 2004).

A confiança baseada nas intenções da marca é fundamentada na crença de que a marca colocará os interesses do consumidor à frente dos seus próprios, em situações que envolverem problemas com o consumo dos produtos. Esta dimensão revela um elemento de seguridade emocional da parte dos indivíduos, baseado na crença do consumidor de que o comportamento da marca é motivado por intenções favoráveis e positivas em relação ao seu bem-estar e interesses, mesmo se houver problemas futuros com os produtos. Esta abordagem envolve a convicção e as ações do consumidor acerca de situações presentes, com base na expectativa confiante de que os acontecimentos futuros provarão estarem corretos, ou seja, de que a marca não tirará vantagem da vulnerabilidade do consumidor. Dessa forma, esta dimensão envolve aspectos como o altruísmo, benevolência, honestidade e justiça (DelgadoBallester, 2004).

Delgado-Ballester, Munuera-Aleman e Yague-Guillen (2003) desenvolveram e validaram uma escala de confiança na marca, que é aplicada em um estudo posterior em duas categorias de produto distintas: desodorante e cerveja (Delgado-Ballester, 2004). A pesquisa empírica confirmou a consistência e validade da escala, bem como a existência das duas principais dimensões da confiança na 
marca - a confiabilidade da marca e as intenções da marca. Segundo os achados do estudo, a dimensão da confiabilidade da marca está relacionada com o desempenho da marca e, dessa forma, abarca as características funcionais e os atributos físicos do produto. A dimensão das intenções da marca compreende as crenças e significados sobre a marca que extrapolam as suas funções físicas, estando mais relacionada com aspectos da personalidade da marca, como honestidade e sinceridade.

Considerando-se a influência da confiança na marca nos processos de adoção de novas tecnologias, entende-se que a relação de confiança previamente estabelecida entre o consumidor e a marca, a qual é baseada na confiabilidade e nas intenções da marca endossante, transmitirá informações para o consumidor acerca da nova tecnologia que está sendo lançada, reduzindo a assimetria de informações e atuando como um sinalizador da qualidade do produto. Dessa forma, a confiança na marca, por meio das suas duas dimensões, possibilitará a redução do risco percebido pelo consumidor nestes processos, influenciando positivamente a sua intenção de adotar esta nova tecnologia.

Com base na discussão acima, define-se a seguinte hipótese:

H1: A confiança baseada na (a) confiabilidade e (b) nas intenções da marca endossante terá uma influência positiva na intenção do consumidor de adotar uma nova tecnologia.

\subsection{O UNIFIED THEORY OF ACCEPTANCE AND USE OF TECHNOLOGY (UTAUT)}

Na tentativa de unificar as teorias mais utilizadas referentes à aceitação e ao uso da Tecnologia da Informação (TI), Venkatesh et al. (2003) exploraram as oito teorias mais relevantes na literatura de adoção de novas tecnologias, identificando as principais dimensões e operacionalização destas. Estes autores realizaram uma survey em quatro organizações que já haviam adquirido uma determinada TI e que estavam introduzindo-a aos usuários, para testar quais destas dimensões seriam realmente relevantes. Apesar de esta pesquisa ter sido realizada num contexto de adoção de uma tecnologia por um profissional em uma empresa e não por um consumidor que tenha feito a livre escolha de adoção por determinado produto ou serviço, considerou-se adequada a utilização deste mesmo modelo, pelo fato de que os fatores que influenciam a intenção de adoção de uma nova tecnologia podem ser, pelo menos em

parte, semelhantes àqueles encontrados nos estudos sumarizados por Venkatesh et al. (2003). É importante ressaltar que, a fim de que esta adaptação fosse feita, todas as medidas utilizadas, bem como o contexto de aplicação da pesquisa, foram reorientados para as situações de consumo de tecnologia habituais dos consumidores.

Revista de Administração e Inovação, São Paulo, v. 7, n. 4, p.162-185, out./dez. 2010 
O teste do modelo dos autores confirmou que a expectativa de desempenho, a expectativa de esforço e a influência social possuem impacto significativo na intenção de comportamento, a qual influencia diretamente no comportamento de uso de uma nova tecnologia, conforme modelo da Figura 1. Assim, o presente estudo partirá dos achados deste trabalho, incluindo a confiança na marca como um elemento adicional a exercer influência neste processo. A Figura 2 apresenta o modelo proposto, o qual consiste no modelo UTAUT acrescido da confiança baseada na confiabilidade (H1a) e nas intenções (H1b) da marca endossante. A seguir, cada um dos três construtos abordados por Venkatesh et al. (2003), bem como a intenção de comportamento, serão analisados.

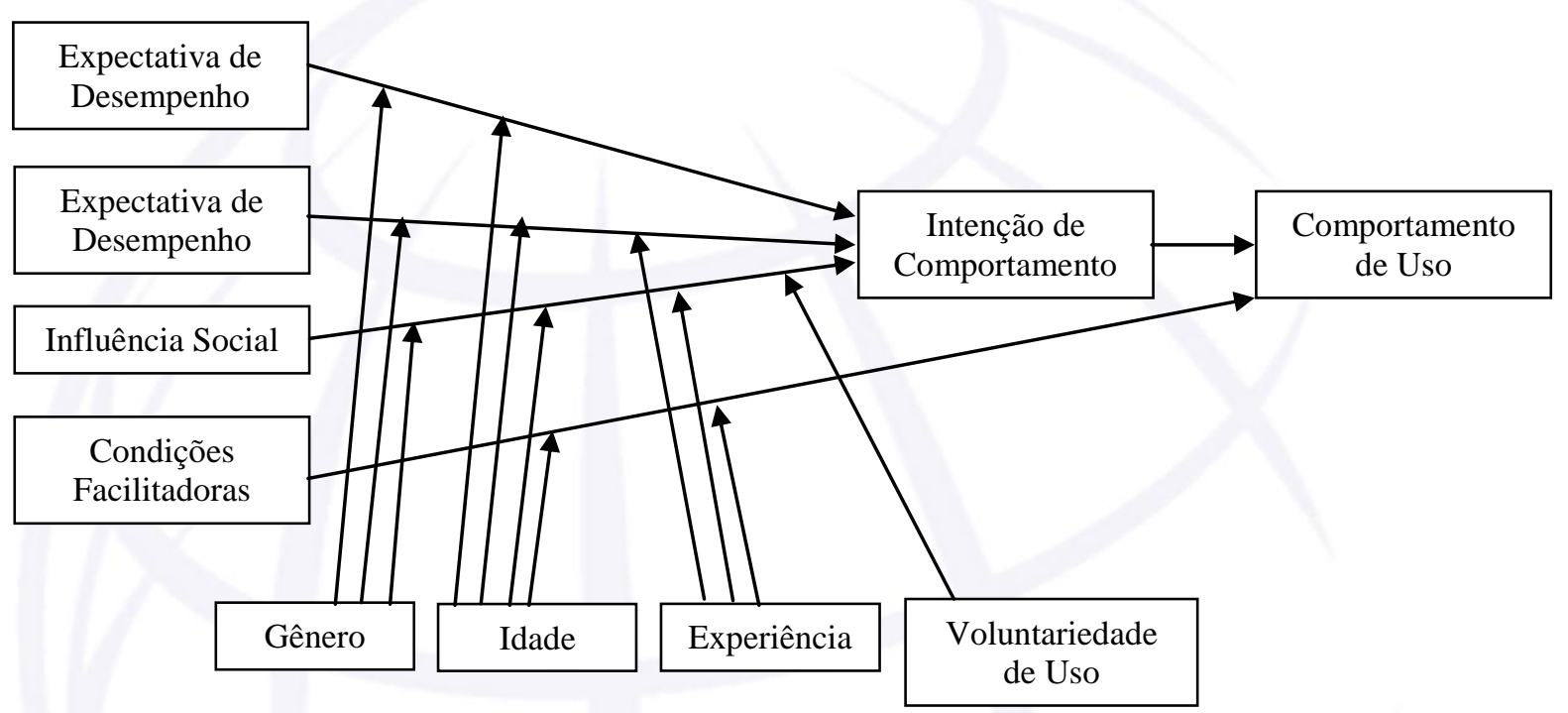

Figura 1: Aceitação da tecnologia da informação pelo usuário.

Fonte: Venkatesh et al., 2003, p. 447.

A expectativa de desempenho pode ser definida como o nível que um indivíduo acredita que o uso da tecnologia irá ajudá-lo a alcançar ganhos no seu trabalho (Venkatesh et al., 2003). No caso deste estudo, o conceito foi ampliado (assim como as medidas adaptadas) para abranger os ganhos de desempenho que o consumidor irá obter em suas atividades diversas. A expectativa desse cumprimento foi construída a partir de cinco construtos já existentes (Venkatesh et al, 2003): utilidade percebida (Davis, 1989; Davis, Bagozzi, \& Warshaw, 1989), motivação extrínseca (Davis, Bagozzi, \& Warshaw, 1992), ajuste ao trabalho (Thompson et al., 1991), vantagem relativa (Moore \& Benbasat, 1991) e expectativa de resultado (Compeau \& Higgins, 1995; Compeau et al., 1999).

A expectativa de esforço é definida como o grau de facilidade associado ao uso do sistema (Venkatesh et al., 2003). No caso do estudo do comportamento do consumidor, seria o grau de 
facilidade que o consumidor associa ao uso de um determinado produto ou serviço. Para a mensuração do construto foram utilizados basicamente três modelos existentes para a expectativa de esforço (Venkatesh et al., 2003): a facilidade de uso percebida (Davis, 1989; Davis et al., 1989); a complexidade (Thompson, Higgins, \& Howell, 1991) e a facilidade de uso (Moore \& Benbasat, 1991).

A influência social é definida como o grau no qual um indivíduo percebe que os outros acham importante utilizar determinado sistema. Para a mensuração da influência social, Venkatesh et al. (2003) basearam-se nos seguintes temas: norma subjetiva (Azjen, 1991; Davis et al., 1989; Fishbein \& Azjen, 1975; Mathieson, 1991; Taylor \& Todd, 1995a; 1995b), fatores sociais (Thompson et al., 1991) e imagem (Moore \& Benbasat, 1991).

Por fim, a intenção de comportamento consiste na intenção do usuário em relação ao uso efetivo do sistema e é um importante antecedente do comportamento de uso efetivo pelo indivíduo (Venkatesh et al., 2003).

A partir das informações levantadas, as demais hipóteses são definidas:

H2: A expectativa de desempenho terá uma influência positiva na intenção do consumidor de adotar uma nova tecnologia.

H3: A expectativa de esforço terá uma influência negativa na intenção do consumidor de adotar uma nova tecnologia.

H4: A influência social terá uma influência positiva na intenção do consumidor de adotar uma nova tecnologia. 


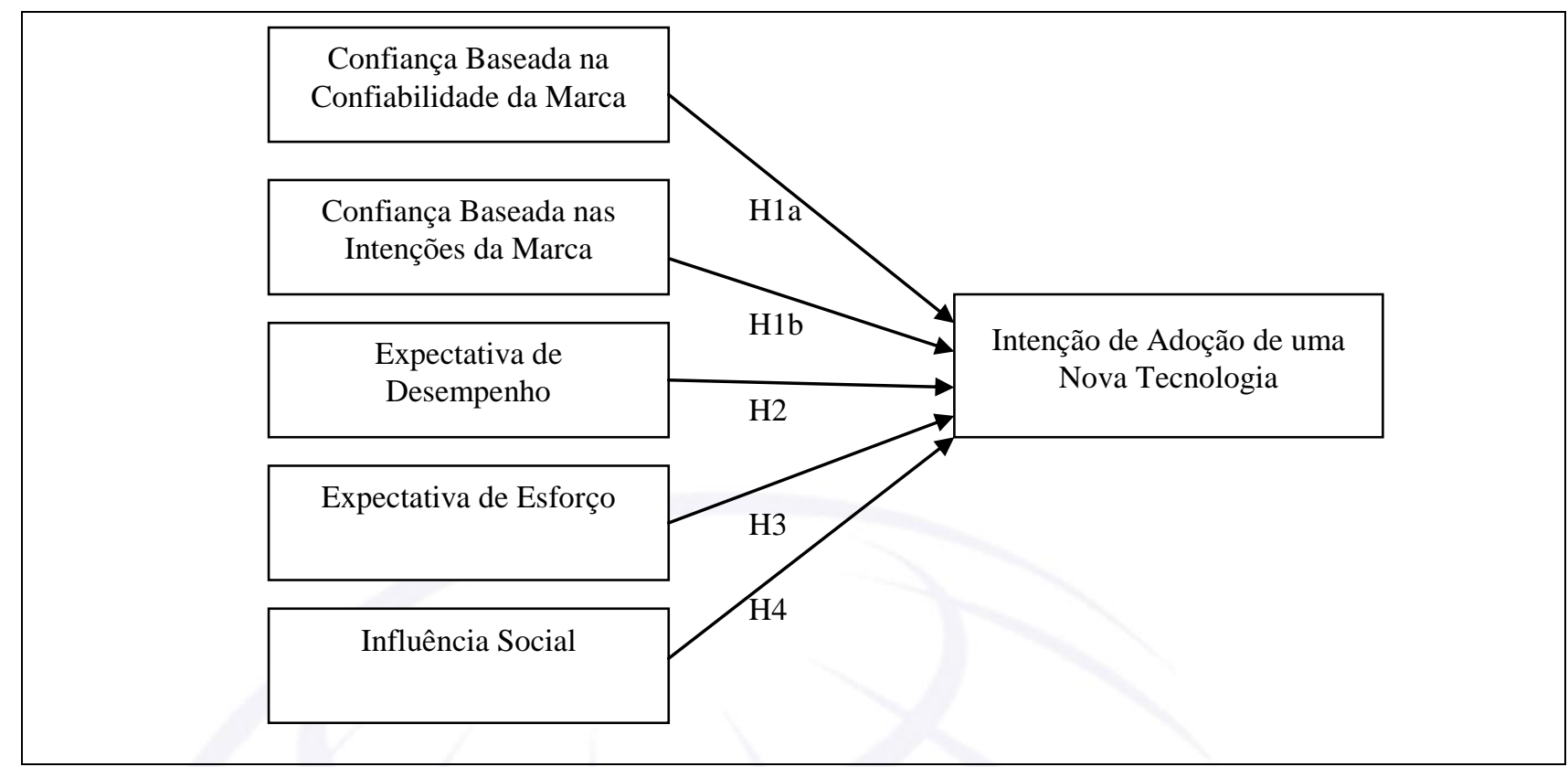

Figura 2 - Antecedentes da intenção do consumidor na adoção de novas tecnologias.

Fonte: Elaborado pelos autores.

\section{MÉTODO}

A fim de atender ao objetivo definido para o presente trabalho, foi realizada uma pesquisa de natureza descritiva, na qual foi empregado o método survey. Na aplicação da pesquisa foi apresentada aos respondentes uma nova tecnologia, que consistia em um computador portátil no formato de um conjunto de canetas que projetavam o monitor, teclados e periféricos, que desempenhava todas as funções normais de um computador. O pesquisador enfatizava, no momento da explicação, que se tratava de uma tecnologia em fase de protótipo que estava sendo desenvolvida por um conhecido fabricante de produtos tecnológicos. A fim de preservar a identidade da marca em questão, optou-se por denominá-la neste artigo de "TEC". A tecnologia era apresentada aos alunos por meio de slides, os quais simulavam o web site da marca, respeitando a sua identidade visual, de modo a parecer que o produto estava sendo demonstrado no próprio web site da empresa.

A amostra dos respondentes foi composta por 348 alunos de cursos de graduação de duas instituições de ensino superior da Região Metropolitana de Porto Alegre, que apresentavam as seguintes características: idade entre 17 e 50 anos (sendo que 68,8\% dos pesquisados possuíam idade entre 18 e 25 anos), de 2 a 20 anos de experiência com computadores (sendo que 68,8\% afirmaram ter entre 8 e 13

Revista de Administração e Inovação, São Paulo, v. 7, n. 4, p.162-185, out./dez. 2010 
anos de experiência) e posse de computador no domicílio (97,1\% dos pesquisados). Após a apresentação da tecnologia, solicitou-se aos pesquisados que respondessem ao instrumento de pesquisa, que continha uma escala que avaliava a intenção de comportamento do respondente em adotar a tecnologia que estava sendo apresentada e outra escala que avaliava a confiança do respondente na marca que supostamente havia desenvolvido esta tecnologia.

Os instrumentos (tanto o de confiança na marca, extraído de Delgado-Ballester [2004], quanto o de adoção de tecnologia, adaptado de Venkatesh et al. [2003]), originalmente na língua inglesa, passaram por tradução para a língua portuguesa, validação por pesquisadores que já trabalhavam no tema e tradução reversa para a língua inglesa, a fim de assegurar que os termos possuíam significados semelhantes nos dois idiomas. Adicionalmente, para assegurar a compreensão do que se pretendia medir, foram conduzidos dois pré-testes com alunos, ocasião na qual se apresentou a tecnologia e os instrumentos, para, posteriormente, questioná-los sobre a compreensão de cada item. Este processo permitiu que pequenos ajustes fossem feitos, até chegar-se à versão final dos instrumentos, nos quais foram utilizadas escalas de 1 (discordo fortemente) a 7 (concordo fortemente).

\subsection{MEDIDAS}

A medida da intenção de comportamento em adotar a nova tecnologia foi feita com base na escala concebida e validada por Venkatesh et al. (2003). A intenção de comportamento, que é objeto de estudo do presente trabalho, consiste em uma etapa intermediária do modelo, influenciando diretamente o comportamento de uso e sendo influenciada diretamente por três construtos: expectativa de desempenho, expectativa de esforço e influência social.

A expectativa de desempenho foi medida por meio das seguintes questões: "eu acharia útil esta tecnologia nas minhas atividades", "utilizar esta tecnologia me possibilita realizar tarefas mais rapidamente", "utilizar esta tecnologia aumentaria minha produtividade" e "se eu utilizar esta tecnologia, eu aumentarei minhas chances de ser mais bem sucedido nas minhas atividades".

A expectativa de esforço foi avaliada por meio das questões "minha interação com esta tecnologia seria clara e compreensível", "seria fácil tornar-me habilidoso na utilização desta tecnologia", "eu acharia esta tecnologia fácil de usar" e "aprender a operar esta tecnologia é fácil para mim".

A influência social foi medida pelas questões: "pessoas que influenciam o meu comportamento pensam que eu deveria usar esta tecnologia", "pessoas que são importantes para mim pensam que eu

Revista de Administração e Inovação, São Paulo, v. 7, n. 4, p.162-185, out./dez. 2010 
deveria usar esta tecnologia", "as pessoas que são importantes para mim poderiam ser úteis na utilização desta tecnologia" e "em geral, receberia apoio para utilização da tecnologia".

A intenção de comportamento foi medida por meio das seguintes questões: "eu pretendo utilizar a tecnologia nos próximos 12 meses se ela estivesse disponível no mercado", "eu prevejo que utilizarei a tecnologia nos próximos 12 meses se ela estivesse disponível no mercado" e "eu planejo utilizar a tecnologia nos próximos 12 meses se ela estivesse disponível no mercado”.

A confiança na marca foi medida por meio da escala desenvolvida e validada por DelgadoBallester (2004). Assim, o instrumento contemplava as duas dimensões da confiança na marca, sendo que as quatro primeiras questões referiam-se à confiabilidade da marca e as quatro questões seguintes às intenções da marca. Dessa forma, o instrumento contava com as seguintes questões: "TEC é uma marca que atende as minhas expectativas", "eu sinto confiança na marca TEC", "TEC é uma marca que nunca me desaponta", "a marca TEC garante satisfação", "TEC seria honesta e sincera ao se dirigir a meus interesses", "eu poderia contar com a marca TEC para resolver um problema", "a marca TEC faria qualquer esforço para me satisfazer", "a marca TEC me compensaria de alguma forma por problemas com o produto".

\section{RESULTADOS}

Primeiramente será apresentada a preparação dos dados, após a análise fatorial confirmatória, confiabilidade e validade discriminante. Por fim, no último subitem, será apresentado o teste das hipóteses.

\subsection{PREPARAÇÃO DOS DADOS}

Antes de iniciar a análise dos dados, foi realizada uma preparação para que fossem tratados os casos omissos, observações atípicas, bem como verificada a linearidade, a normalidade e a homocedasticidade da variância no Software SPSS 14.0. Tabachnick e Fidell (2001) apontam que esta etapa é essencial para garantir a validade dos pressupostos das análises e, consequentemente, dos resultados. Após, foi feita a análise fatorial confirmatória e verificada a confiabilidade e a validade discriminante das medidas. Por fim, os dados foram analisados através de Modelagem de Equações Estruturais, considerando-se, para tal, a intenção de comportamento como variável dependente e as 
variáveis de confiança na marca (nas duas dimensões distintas, formadas pela confiabilidade e intenções da marca), expectativa de desempenho, expectativa de esforço e influência social como variáveis independentes.

Primeiramente, iniciou-se a preparação dos dados através da análise dos casos omissos. Foram encontrados 19 casos e optou-se por retirá-los. As observações atípicas (outliers) foram detectadas de forma univariada através do Teste $\mathrm{Z}$ e, de forma multivariada, utilizando a distância de Mahalanobis. Para o teste de normalidade, foi realizado o teste de assimetria e curtose onde todos os valores encontrados foram considerados satisfatórios.

O teste da homoscedasticidade foi feito utilizando a Estatística de Levene. Foram empregadas todas as variáveis métricas e as variáveis não-métricas "gênero" e "renda". Observou-se que as variáveis que apresentaram heteroscedasticidade não eram as mesmas ao longo das variáveis "gênero" e "renda". Nesse caso, conforme Hair, Anderson, Tatham e Black (2005), não é necessária nenhuma alteração, a não ser que os casos fossem os mesmos ao longo das diferentes variáveis não-métricas. O teste de linearidade foi realizado e todas as variáveis apresentaram linearidade, não sendo necessária a retirada de nenhuma questão.

\subsection{ANÁLISE FATORIAL CONFIRMATÓRIA (AFC), CONFIABILIDADE E VALIDADE DISCRIMINANTE}

Após a preparação dos dados, os mesmos foram migrados para o software LISREL 8.0 para que fosse realizada a Análise Fatorial Confirmatória. A primeira análise buscou verificar a aderência das variáveis aos construtos de primeira ordem a elas relacionados pela teoria. Apresentam-se, na sequência, os resultados obtidos na análise fatorial confirmatória da confiança na marca, expectativa de desempenho, expectativa de esforço e influência social.

A Análise Fatorial Confirmatória foi realizada para cada um dos construtos e as questões CM8 (“A marca TEC me compensaria de alguma forma por problemas com o produto") e IS4 ("Em geral, receberia apoio para utilização da tecnologia") necessitaram ser retiradas devido aos erros serem maiores que as cargas das variáveis, conforme pode ser observado na Tabela 1 (em negrito). Os índices de ajuste do modelo de medidas foram satisfatórios com índices superiores a 0.90 (Hair et al., 2005).

Revista de Administração e Inovação, São Paulo, v. 7, n. 4, p.162-185, out./dez. 2010 


\begin{tabular}{|c|c|c|c|}
\hline Relação & $\begin{array}{l}\text { Valor } \\
\text { Padronizado }\end{array}$ & Erro Padrão & Valor total \\
\hline $\mathrm{CM} 1 \rightarrow \mathrm{CM} 1$ & 0,71 & 0,49 & 14,23 \\
\hline $\mathrm{CM} 1 \rightarrow \mathrm{CM} 2$ & 0,76 & 0,43 & 15,51 \\
\hline $\mathrm{CM} 1 \rightarrow \mathrm{CM} 3$ & 0,79 & 0,37 & 16,56 \\
\hline $\mathrm{CM} 1 \rightarrow \mathrm{CM} 4$ & 0,91 & 0,17 & 20,25 \\
\hline $\mathrm{CM} 2 \rightarrow \mathrm{CM} 5$ & 0,84 & 0,29 & 17,60 \\
\hline $\mathrm{CM} 2 \rightarrow \mathrm{CM} 6$ & 0,87 & 0,25 & 18,35 \\
\hline $\mathrm{CM} 2 \rightarrow \mathrm{CM} 7$ & 0,73 & 0,47 & 14,58 \\
\hline CM2 $\rightarrow$ CM8 & 0,60 & 0,64 & 11,24 \\
\hline $\mathrm{ED} \rightarrow \mathrm{ED} 1$ & 0,72 & 0,48 & 14,33 \\
\hline $\mathrm{ED} \rightarrow \mathrm{ED} 2$ & 0,84 & 0,30 & 17,72 \\
\hline ED $\rightarrow$ ED3 & 0,85 & 0,28 & 18,05 \\
\hline $\mathrm{ED} \rightarrow \mathrm{ED} 4$ & 0,75 & 0,44 & 15,19 \\
\hline $\mathrm{EE} \rightarrow \mathrm{EE} 1$ & 0,70 & 0,51 & 14,13 \\
\hline $\mathrm{EE} \rightarrow \mathrm{EE} 2$ & 0,73 & 0,47 & 14,94 \\
\hline $\mathrm{EE} \rightarrow \mathrm{EE} 3$ & 0,91 & 0,17 & 20,60 \\
\hline $\mathrm{EE} \rightarrow \mathrm{EE} 4$ & 0,89 & 0,20 & 20,04 \\
\hline IS $\rightarrow$ IS1 & 0,86 & 0,26 & 18,97 \\
\hline IS $\rightarrow$ IS2 & 0,98 & 0,03 & 23,53 \\
\hline IS $\rightarrow$ IS3 & 0,70 & 0,51 & 14,20 \\
\hline IS $\rightarrow$ IS4 & 0,59 & 0,65 & 11,53 \\
\hline $\mathrm{IC} \rightarrow \mathrm{IC} 1$ & 0,84 & 0,29 & 18,38 \\
\hline $\mathrm{IC} \rightarrow \mathrm{IC} 2$ & 0,94 & 0,12 & 21,75 \\
\hline $\mathrm{IC} \rightarrow \mathrm{IC} 3$ & 0,87 & 0,24 & 19,33 \\
\hline
\end{tabular}

Tabela 1 - Estatísticas da AFC.

Fonte: Elaborado pelos autores.

Após a realização dos devidos ajustes da Análise Fatorial Confirmatória, foi auferida a confiabilidade das medidas, bem como a validade discriminante. Para tal, foi utilizado o teste proposto por Fornell e Larcker (1981), o qual afirma que a variância extraída para cada construto deve ser maior que o quadrado da correlação (Tabela 2) entre o construto e qualquer outro construto, que caracteriza a variância compartilhada. Este teste apresentou valores satisfatórios, conforme apresentado na Tabela 3, a seguir. 


\begin{tabular}{crrrrrr}
\hline & ED & EE & IS & IC & CM1 & CM2 \\
\hline ED & 1 & & & & & \\
\hline EE & 0,30 & 1 & & & & \\
\hline IS & 0,60 & 0,32 & 1 & & & \\
\hline IC & 0,47 & 0,32 & 0,45 & 1 & & \\
\hline CM1 & 0,21 & 0,21 & 0,15 & 0,21 & & 1 \\
\hline CM2 & 0,31 & 0,20 & 0,28 & 0,32 & 0,73 & 1 \\
\hline
\end{tabular}

Tabela 2 - Matriz de Correlação.

Fonte: Elaborado pelos autores.

Tabela 3 - Validade Discriminante

\begin{tabular}{ccccccc}
\hline & ED & EE & IS & IC & CM1 & CM2 \\
\hline ED & 0,7491 & & & & & \\
\hline EE & 0,0900 & 0,7743 & & & & \\
\hline IS & 0,3600 & 0,1024 & 0,7447 & & & \\
\hline IC & 0,2209 & 0,1024 & 0,2025 & 0,8701 & & \\
\hline CM1 & 0,0441 & 0,0441 & 0,0225 & 0,0441 & 0,7532 & \\
\hline CM2 & 0,0961 & 0,0400 & 0,0784 & 0,1024 & 0,5329 & 0,7104 \\
\hline
\end{tabular}

\section{Tabela 3 - Validade Discriminante}

Fonte: Elaborado pelos autores.

Após o teste do modelo de medidas, o modelo estrutural foi testado no Software LISREL 8.1, a fim de que as hipóteses fossem testadas através de Modelagem de Equações Estruturais.

\subsection{TESTE DAS HIPÓTESES}

A Modelagem de Equações Estruturais permite que os processos causais estudados sejam representados por uma série de equações estruturais, que pode ser modelada através de uma figura que permite a clara visualização da teoria proposta. Assim, o modelo hipotetizado pode ser testado estatisticamente através de uma análise simultânea de todo o sistema de variáveis, determinando em que extensão ele é consistente com os dados (Byrne, 1994). A ideia central proposta no modelo deste trabalho é examinar a influência da confiança na marca, da expectativa de desempenho, da expectativa de esforço e da influência social na intenção de adoção de uma nova tecnologia de uma marca específica.

Primeiramente, foi testado o modelo completo, tendo como variáveis independentes a expectativa

Revista de Administração e Inovação, São Paulo, v. 7, n. 4, p.162-185, out./dez. 2010 
de desempenho (ED), a expectativa de esforço (EF), a influência social (IS), a confiança na marca baseada na confiabilidade (CM1) e a confiança na marca baseada nas intenções (CM2), e como variável dependente a intenção de comportamento (IC). Todavia, nesse teste de modelo, ambas as confianças (CM1 e CM2) tiveram valores " $\mathrm{t}$ " não significativos (-0,30 e 1,90, respectivamente).

Após esse teste de modelo inicial, foi retirada a dimensão da confiança baseada na confiabilidade (CM1), a fim de verificar se a confiança baseada nas intenções (CM2) teria impacto significativo. Esta opção se justifica pelo fato das duas dimensões da confiança na marca, embora apresentem relação uma com a outra, são independentes em relação ao construto geral. Os resultados demonstraram que o impacto da confiança na marca baseada nas intenções (CM2) possui impacto positivo e significativo $(\gamma=$ $0,15 ; \mathrm{t}$-value $=3,78)$. Adicionalmente, foi testado o modelo sem a confiança na marca baseada nas intenções (CM2) e o impacto da confiança baseada na confiabilidade (CM1) apontou um valor "t" não significativo $(\mathrm{t}$-value $=1,68)$. Na Figura 3 , a seguir, é apresentado o modelo estrutural final do presente estudo.

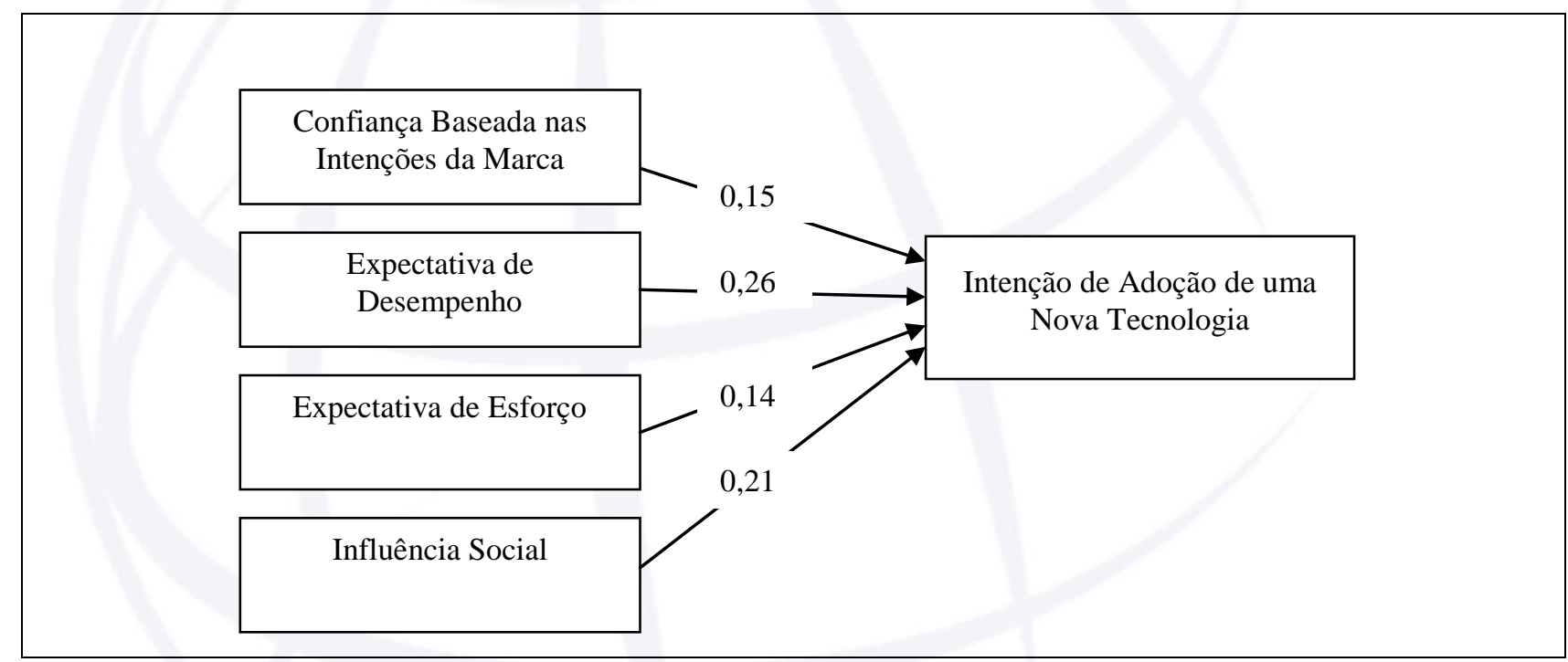

Figura 3 - Modelo estrutural (Cargas Padronizadas).

Fonte: Elaborado pelos autores.

Na Tabela 4 são apresentadas as estatísticas do modelo. Primeiramente são apresentados os parâmetros estimados, seguidos do valor padronizado e do erro padrão e, por fim, o valor t. Todas as relações foram significativas a um nível de significância de 0,01 . 


\begin{tabular}{l|c|c}
\hline \multicolumn{1}{c|}{ Relação } & $\begin{array}{c}\text { Valor } \\
\text { Padronizado }\end{array}$ & Valor t* \\
\hline $\mathrm{CM} 2 \rightarrow \mathrm{IC}$ & 0,15 & 3,78 \\
\hline $\mathrm{ED} \rightarrow \mathrm{IC}$ & 0,26 & 2,53 \\
\hline $\mathrm{EE} \rightarrow \mathrm{IC}$ & 0,14 & 3,09 \\
\hline $\mathrm{IS} \rightarrow \mathrm{IC}$ & 0,21 & 2,65 \\
\hline \multicolumn{2}{l}{}
\end{tabular}

Tabela 4 - Estatísticas do Modelo.

Fonte: Elaborado pelos autores.

A Tabela 5 exibe os índices de ajuste do modelo geral de estudo, verificando-se um ajustamento adequado dos dados ao modelo teórico conforme Hair et al. (2005) e Kline (1998). O único ajuste que apresentou valor pouco abaixo do recomendado foi o GFI $(0,87)$; todavia, conforme Hair et al. (2005), valores acima de 0,80 , no limite, podem ser aceitos.

\begin{tabular}{c|c|c|c|c|c|c|c}
\hline & $\chi^{2}$ & GL & $\chi^{2 / G L}$ & NFI & GFI & CFI & RMSEA \\
\hline Modelo Gerado & 292,33 & 115 & 2,54 & 0,92 & 0,87 & 0,95 & 0,08 \\
\hline Valores recomendados & & & $<3$ & $>0,9$ & $>0,9$ & $>0,9$ & $0,04 \sim 0,08$ \\
\hline
\end{tabular}

Tabela 5 - Índices de Ajuste do Modelo Estrutural Completo.

Fonte: processamento dos dados; valores desejáveis de Hair et al., 2005, e Kline, 1998.

Ao testar a relação postulada na hipótese 1 de que "a confiança baseada na (a) confiabilidade e (b) nas intenções da marca endossante terá uma influência positiva significante na intenção do consumidor de adotar uma nova tecnologia" verifica-se que a intenção de comportamento é influenciada positivamente $(\gamma=0,15)$ pela confiança baseada nas intenções da marca $(\mathrm{H} 1 \mathrm{~b})$. Todavia, o impacto da confiança baseado na confiabilidade da marca (H1a) não é significativo, portanto a hipótese 1 é parcialmente confirmada. Isso significa dizer que o consumidor estará mais disposto a adotar uma nova tecnologia quando acreditar que a marca é honesta, sincera e esforçada no sentido de resolver os problemas que porventura acontecerem com o produto. Por outro lado, a confiabilidade nos atributos físicos e no desempenho do produto não exerce influência significativa na intenção do consumidor de adotar uma nova tecnologia.

A hipótese 2, que postula que "a expectativa de desempenho terá uma influência positiva significante na intenção do consumidor de adotar uma nova tecnologia”, também foi confirmada. O

Revista de Administração e Inovação, São Paulo, v. 7, n. 4, p.162-185, out./dez. 2010 
impacto da expectativa de desempenho foi o maior quando comparado ao outros construtos examinados $(\gamma=0,26)$. Quanto mais o consumidor acha a tecnologia útil, mais ele acredita conseguir realizar tarefas rapidamente. Assim, o fato de ele tornar-se mais produtivo e de ter chance de ser mais bem sucedido em suas atividades influenciará positivamente a sua intenção de adoção de uma nova tecnologia lançada por uma determinada marca.

A relação proposta na hipótese 3 , de que "a expectativa de esforço terá uma influência negativa significante na intenção do consumidor de adotar uma nova tecnologia" também foi confirmada $(\gamma=$ 0,14). Ou seja, quanto menor a expectativa do consumidor em relação ao esforço na utilização de uma nova tecnologia, em outras palavras, a sua percepção de que terá uma boa interação, que será fácil de operá-la e tornar-se habilidoso na utilização da mesma, maior será a intenção de adoção dessa nova tecnologia.

A hipótese 4, que afirma que "a influência social terá uma influência positiva significante na intenção do consumidor de adotar uma nova tecnologia", também foi confirmada $(\gamma=0,21)$. Isso confirma que a opinião e o suporte das pessoas que são importantes e que influenciam o comportamento do consumidor é um antecedente da intenção de adoção de uma nova tecnologia de uma determinada marca.

\begin{tabular}{l|c}
\hline \multicolumn{1}{c|}{ Hipóteses } & Resultado \\
\hline $\begin{array}{l}\text { H1: A confiança baseada na (a) confiabilidade e (b) nas intenções da marca endossante } \\
\text { terá uma influência positiva na intenção do consumidor de adotar uma nova tecnologia. }\end{array}$ & $\begin{array}{c}\text { Parcialmente } \\
\text { Confirmada }\end{array}$ \\
\hline $\begin{array}{l}\text { H2: A expectativa de desempenho terá uma influência positiva na intenção do } \\
\text { consumidor de adotar uma nova tecnologia }\end{array}$ & Confirmada \\
\hline $\begin{array}{l}\text { H3: A expectativa de esforço terá uma influência negativa na intenção do consumidor } \\
\text { de adotar uma nova tecnologia. }\end{array}$ & Confirmada \\
\hline $\begin{array}{l}\text { H4: Quanto maior a influência social para que o consumidor adote uma nova } \\
\text { tecnologia, maior será a sua intenção de adotá-la. }\end{array}$ & Confirmada \\
\hline
\end{tabular}

\section{Quadro 1: Resumo do Teste de Hipóteses.}

Fonte: Elaborado pelos autores.

\section{CONCLUSÕES}

A velocidade com que novas tecnologias são desenvolvidas e que produtos são lançados no mercado torna necessário um conhecimento mais aprofundado acerca do comportamento do consumidor em relação à adoção destas tecnologias, bem como dos fatores que influenciam neste processo. $\mathrm{O}$ presente estudo demonstrou que a confiança na marca, e mais especificamente a confiança baseada nas 
intenções da marca, tem um impacto significativo neste processo, influenciando na tomada de decisão dos consumidores em adotar uma nova tecnologia.

O fato da confiança baseada nas intenções, e não na confiabilidade da marca, ter sido significativa estimula que algumas análises sejam realizadas a este respeito. A dimensão da confiança baseada na confiabilidade da marca está relacionada ao desempenho e a competência técnica do produto, ou seja, à crença de que o produto irá preencher as expectativas do consumidor. No caso de uma nova tecnologia, o consumidor não conhece totalmente os atributos do produto, e, portanto, as suas expectativas em relação ao seu desempenho não estão bem definidas. Portanto, estas expectativas parecem não ser suficientes para fundamentar a dimensão da confiança baseada na confiabilidade da marca.

Por outro lado, a confiança baseada nas intenções da marca remete a um comportamento futuro da empresa, de que ela priorizará os interesses do consumidor, e não os seus próprios, se surgirem problemas relacionados ao consumo do produto. No caso da aquisição de uma nova tecnologia, o desconhecimento do produto faz com que a incerteza em relação a problemas futuros seja muito grande. Assim, entende-se que a conduta futura da empresa é o elemento de incerteza preponderante para o consumidor, fundamentando a relação de confiança na marca que se estabelece entre ele e a empresa nos processos de adoção de novas tecnologias.

Os achados deste trabalho trazem importantes implicações teóricas e gerenciais. Do ponto de vista teórico, dois pontos merecem destaque. Em primeiro lugar, a inclusão do fator confiança na marca adiciona um novo construto ao modelo UTAUT, o qual previa inicialmente apenas o impacto da expectativa de performance, expectativa de esforço e influência social na intenção de comportamento. Em segundo lugar, a aplicação do modelo, até então restrito ao ambiente profissional, ao contexto de consumo, considerando o consumidor que adota uma tecnologia por motivação e interesse próprios, traz uma nova perspectiva ao modelo. Assim, o presente trabalho amplia o conhecimento teórico existente acerca da adoção de novas tecnologias, ratificando a importância dos três construtos já existentes no modelo e agregando um novo e importante elemento.

A análise dos resultados do ponto de vista gerencial também evidencia considerações importantes. Em primeiro lugar, a confirmação de que a confiança na marca baseada nas intenções da marca é um dos antecedentes da intenção de comportamento nos processos de adoção de novas tecnologias estimula as empresas da área a cultivarem relacionamentos de longo prazo com os seus clientes e a investirem em marcas fortes e confiáveis. Nesse sentido, são recomendados investimentos em comunicação, ressaltando os valores de altruísmo, benevolência e honestidade, os quais estão 
relacionados à dimensão da confiança que foi significante no modelo. Similarmente, ações de marketing que estimulem a percepção do consumidor acerca da disponibilidade da marca, em especial frente à possibilidade de problemas futuros, também devem ser priorizadas. Por fim, é fundamental que as empresas desenvolvam ações de resposta eficazes aos problemas que eventualmente surgirem no lançamento de seus produtos. Embora o setor tecnológico tenha características específicas, como a inovação constante e o lançamento de novos produtos, o estudo deixa claro que as práticas tradicionais de marketing não devem ser negligenciadas neste segmento. No próximo subitem serão apresentadas as limitações do estudo e sugestões para futuras pesquisas.

\subsection{LIMITAÇÕES E SUGESTÕES PARA PESQUISAS FUTURAS}

As contribuições do presente estudo são ponderadas pelas limitações que o circundam e abrem possibilidades para novas pesquisas. Uma das limitações se refere à utilização de uma marca de grande confiabilidade junto aos pesquisados, o que pode ter limitado os resultados do estudo neste sentido. Portanto, sugere-se que futuras pesquisas utilizem marcas desconhecidas e de pouca confiabilidade para verificar se os resultados são replicados.

A análise do modelo de adoção de novas tecnologias sob a perspectiva da economia da informação, no qual a marca exerce a função de reduzir a incerteza e o risco subjacentes a estes processos, estimula a definição de novos questionamentos. Por exemplo, com base em estudos realizados sobre as garantias (Wiener, 1985; Boulding \& Kirmani, 1993), o preço (Gerstner, 1985; Rao \& Monroe, 1989), e a propaganda (Kirmani \& Wright, 1989; Kirmani, 1990, 1997; Barone, Taylor, \& Urbany; 2005), cujos resultados demonstraram a eficácia destes fatores na sinalização da qualidade, novas pesquisas poderiam avaliar o papel destes elementos na redução do risco e da incerteza nos processos de adoção de novas tecnologias. De forma similar, poderia ser analisado o potencial da confiança na marca na redução da incerteza e do risco em outros processos de consumo, como serviços relacionados ao conhecimento (cursos universitários, por exemplo) e saúde (serviços hospitalares e exames, por exemplo). Além disso, o efeito moderador de fatores como a idade, gênero, renda e experiência de uso, dentre outros, poderia ser avaliado, a fim de que se pudesse determinar se o perfil dos consumidores influencia na relação entre os diversos construtos.

Assim, embora ainda haja diversas questões em aberto acerca deste tema, o objetivo principal deste trabalho foi atingido. Os resultados demonstraram que a confiança baseada nas intenções da marca, assim como a expectativa de desempenho, expectativa de esforço e influência social, são 
antecedentes na adoção de novas tecnologias, influenciando a intenção de comportamento do consumidor nestes processos. Uma grande contribuição do trabalho foi buscar através de uma teoria da área de tecnologia da informação encontrar informações que expliquem o comportamento do consumidor na adoção de novas tecnologias que são cada vez mais importantes nos dias de hoje.

\section{REFERÊNCIAS}

Ajzen, I. (1991).The Theory of Planned Behavior. Organizational Behavior and Human Decision Processes, 50(2), 179-211.

Barone, M., Taylor, V., \& Urbany, J. (2005). Advertising signaling effects for new brands: The moderating role of perceived brand differences. Journal of Marketing, 13(1), 1-13.

Byrne, B. M. (1994). Structural equation modeling with LISREL and EQS - Windows: Basic concepts, applications and programming. Thousand Oaks, CA: Sage.

Boulding, W., \& Kirmani, A. (1993). A consumer-side experimental examination of signalling theory. Journal of Consumer Research, 20(1), 111-123.

Chaudhuri, A., \& Holbrook, B. (2001). The chain of effects from brand trust and brand affect to brand performance: The role of brand loyalty. Journal of Marketing, 65(2), 81-93.

Compeau, D. R., Higgins, C. A., \& Huff, S. (1999). Social cognitive theory and individual reactions to computing technology: A longitudinal study. MIS Quarterly, 23(2), 145-158.

Compeau, D. R., \& Higgins, C. A. (1995). Computer self-efficacy: Development of a measure and initial test. MIS Quarterly, 19(2), 189-211.

Crosby, L. A., Evans, K. R., \& Cowles, D. (1990, July). Relationship quality in services selling: An interpersonal influence perspective. Journal of Marketing, 54(3), 68-81.

Davis, F. D. (1989, September). Perceived Usefullness, Perceived easy of use, and user acceptance of information technology. Management Information Systems Quarterly, 13(3), 319-339.

Davis, F. D., Bagozzi, R. P., \& Warshaw, P. R. (1989). User acceptance of computer technology: A comparison of two theoretical models. Management Science, 35(8), 982-1002.

Davis, F. D., Bagozzi, R. P., \& Warshaw, P. R. (1992). Extrinsic and intrinsic motivation to use computers in the workplace. Journal of Applied Social Psychology, 22(14), 1111-1132.

Delgado-Ballester, E. (2004). Applicability of a brand trust scale across product categories: A multigroup invariance analysis. European Journal of Marketing, 38(5/6), 573-592.

Revista de Administração e Inovação, São Paulo, v. 7, n. 4, p.162-185, out./dez. 2010 
Delgado-Ballester, E., \& Munuera-Alemán, J. L. (2005). Does brand trust matter to brand equity? Journal of Product and Brand Management, 14(3), 187-196.

Delgado-Ballester, E., Munuera-Aleman, J. L., \& Yague-Guillen, M. J. (2003). Development and validation of a brand trust scale. International Journal of Market Research, 45(1), 335-353.

Erdem, T., \& Swait, J. (1998). Brand equity as a signaling phenomenon. Journal of Consumer Psychology, 7(2), 131-157.

Erdem, T., Swait, J., \& Valenzuela, A. (2006, January). Brand as signals: A cross-country validation study. Journal of Marketing, 70(1), 34-49.

Fishbein, M., \& Ajzen, I. (1975). Belief, attitude, intention and behavior: An introduction to theory and research. MA: Addison-Wesley, Reading.

Fornell, C., \& Larcker, D. (1981). Structural equation models with unobservable variables and measurement error. Journal of Marketing Research, 18(1), 39-50.

Gerstner, E. (1985, May). Do higher prices signal higher quality? Journal of Marketing Research, 22(2), 209-215.

Hagen, J. M., \& Choe, S. (1998). Trust in Japanese interfirm relations: Institutional sanctions matter. Academy of Management Review, 23(3), 589-600.

Hair, J. F. Jr., Anderson, R. E., Tatham, R. L., \& Black, W. C. (2005). Análise multivariada de dados (5a ed.). Porto Alegre: Bookman.

Kirmani, A., \& Wright, P. (1989). Money talks: Perceived advertising expense and expected product quality. Journal of Consumer Research, 16(3), 344-353.

Kirmani, A. (1990). The effect of perceived advertising costs on brand perceptions. Journal of Consumer Research, 17(2), 160-171.

Kirmani, A. (1997). Advertising repetition as a signal of quality: If it's advertised so often, something must be wrong. Journal of Advertising, 26(3), 77-86.

Kline, R. B. (1998). Principles and practice of structural equation modeling. New York: The Guilford Press.

Lee, B., Ang, L., \& Dubelaar, C. (2005). Lemons on the Web: A Signalling Approach to the Problem of Trust in Internet Commerce. Journal of Economic Psychology, 26(5), 607-623.

Luhmann, N. (1979). Trust and Power. New York: Wiley.

Mathieson, K. (1991). Predicting user intentions: Comparing the technology acceptance model with the theory of planed behavior. Information Systems Research, 2(3), 173-191.

Revista de Administração e Inovação, São Paulo, v. 7, n. 4, p.162-185, out./dez. 2010 
Mishra, A. K. (1996). Organizational responses to crisis: The centrality of trust. In R. M. Kramer \& T. R. Tyler (Eds.). Trust in Organizations: Frontiers of Theory and Research. Thousand Oaks. p. 261287.California: Sage Publications.

Molho, I. (1997). The Economics of Information. Oxford: Blackwell Publishing.

Molm, L. D., Takahashi, N., \& Peterson, G. (2000, March). Risk and trust in social exchange: An experimental test of classic preposition. American Journal of Sociology, 105(5), 1397-427.

Moore, G. C., \& Benbasat, I. (1991, September). Development of an instrument to measure the perceptions of adopting in information technology innovation. Information Systems Research, 2(3), 192222.

Morgan, R.M., \& Hunt, S. D. (1994, July). The Commitment-Trust theory of relationship marketing. Journal of Marketing, 58, p. 20-38.

Njite, D., \& Parsa, H. G. (2005, Abril/September). Structural equation modeling of factors that influence consumer internet purchase intentions services. Journal of Services Research, 5(1), 43-59.

Pavlou, P. A. (2003, spring). Consumer acceptance of eletronic commerce: Integrating trust and risk with the technology acceptance model. International Journal of Electronic Commerce, 7(3), 101-134.

Rao, A. R., \& Monroe, K. B. (1989, August). The effect of price, brand name, and store name on buyers' perception of product quality: An integrative review. Journal of Marketing Research, 26(3), 351-357.

Rogers, E. M. (1995). Diffusion of Innovations. New York: The Free Press.

Rosseau, D. M., Sitkin, S. B., Burt, R. S., \& Camerer, C. (1998). Not so different after all: A cross discipline view of trust. Academy of Management Review, 23(3), 393-404.

Singh, N., Fassot, G., Chao, M. C. H., \& Hoffmann, J. A. (2006). Understanding international web site usage. International Marketing Review, 2(31), 83-97.

Swan, J. E., Trawick I. E., \& Silva D. W. (1985). How industrial salespeople gain customer trust. Industrial Marketing Management, 1(43), 203-211.

Tabachnick, B. G., \& Fidell, L. S. (2001). Using multivariate statistics. (4th ed.). MA: Allyn \& Bacon, Hardcover, Needham Heights.

Taylor, S., \& Todd, P.A. (1995a). Assessing IT usage: The role of prior experience. MIS Quarterly, 19(2), 85-92.

Taylor, S., \& Todd, P.A. (1995b). Understanding information technology usage: A test of competing models, Information Systems Research, 6(4), p. 144-176.

Revista de Administração e Inovação, São Paulo, v. 7, n. 4, p.162-185, out./dez. 2010 
Thompson, R.L.; Higgins, C.A.; Howell, J. M. (1991). Personal computing: Toward a conceptual model of utilization. MIS Quarterly, 15(1), 124-143.

Venkatesh, V.; Morris, M. G.; Davis, G. B.; Davis, F. D. (2003, September). User aceptance of information technology: Toward a unified view. Management Information Systems Quarterly, 27(3), 425-478.

Wiener, J. R. (1985, September). Are warranties accurate signals of product reliability? Journal of Consumer Research, 12(2), 245-250.

\title{
THE ROLE BRAND LOYALTY PLAYS IN THE INTENTION TO ADOPT NEW
} TECHNOLOGIES

\begin{abstract}
The process of adopting a new technology is characterized by asymmetric information, where the manufacturer is fully aware of the technology's characteristics, but the end-user, to whom this technology is designed, has little access to information. In order to minimize asymmetry, hence reducing the uncertainty and risks inherent in this process, companies can send signals via robust and trusted brands, which may relay information about product quality on to their consumers. This study's principal objective is to analyze the influence of brand loyalty in the adoption process of new technologies in business-to-consumer (B2C) exchanges. Therefore, we applied the Unified Theory of Acceptance and Use of Technology (UTAUT) model, proposed by Venkatesh et al. (2003). Brand loyalty was measured using the scale developed and validated by Delgado-Ballester et al. (2003). Data was collected via a survey administered to 348 respondents, coupled with data analysis performed using Structural Equation Modeling (SEM). The results demonstrated that trust based on brand intentions, which is related to consumer expectations regarding future firm behavior in solving problems associated with the consumption of the product, has significant bearing on the consumer's intention to adopt a new technology. In addition, the constructs previously submitted into the UTAUT model, i.e., social influence, the expectation of i) performance and ii) effort, were confirmed.
\end{abstract}

Keywords: Brand Trust; Consumer Behavior; New Technologies; UTAUT.

Data do recebimento do artigo: 07/08/2010

Data do aceite de publicação: 15/10/2010

Revista de Administração e Inovação, São Paulo, v. 7, n. 4, p.162-185, out./dez. 2010 